\title{
Electron microscopic study of epithelial downgrowth after penetrating keratoplasty
}

\author{
TATSUO YAMAGUCHI, FRANK M. POLACK, AND JOHN VALENTI \\ From the Department of Ophthalmology, College of Medicine, University of Florida, USA
}

SUMMARY An electron microscopic study of 2 cases of epithelial downgrowth after penetrating keratoplasty is presented. Several differences were observed between the normal epithelium and downgrowth epithelium at the ultrastructure level. At the advancing edge of the downgrowth a flattened and fibroblastic endothelium was still present. An interaction area was observed between these 2 tissues. The epithelial downgrowth usually has 5 to 6 layers of cells and either replaces damaged endothelium or destroys it as it advances. The attachment of this downgrowth tissue to the Descemet's membrane at the centre of the graft seemed looser than attachment of a normal epithelium to Bowman's membrane. Near the host-graft junction the downgrowth epithelium, with basement membrane, was tightly attached to a fibrous retrocorneal membrane. Clinical and histological findings suggest that the absence of endothelium assists in the advance of the epithelial downgrowth, but it is not an essential factor. The causes of this downgrowth are thought to be (1) a thin host cornea; (2) incomplete cutting and wound incarceration of Descemet's membrane; and (3) tight sutures with disruption of an old cataract wound.

Epithelial invasion of the anterior chamber is known to occur after cataract surgery and corneal perforations, but it is infrequent after penetrating keratoplasties. ${ }^{1-7}$ The incidence of this complication in histological specimens of repeated corneal grafts has been reported as $4.9 \%{ }^{4}$ or $0.8 \%{ }^{6}$ However, in a study of 1500 keratoplasties Sugar et al. ${ }^{7}$ found an incidence of $0.27 \%$.

This paper describes the ultramicroscopic observations in 2 cases of epithelial downgrowth after penetrating keratoplasty and discusses the probable mechanism of epithelial growth on the posterior corneal surface.

\section{Patients and methods}

\section{CASE REPORTS}

Case 1. A 40-year-old white female underwent a penetrating keratoplasty for corneal scars on her left eye in 1962 and on the right eye in 1963. An uncomplicated intracapsular cataract extraction was performed on her right eye in 1970. Gradual decompensation of both corneal transplants deve-

Correspondence to Frank M. Polack, MD, Department of Ophthalmology, College of Medicine, Box J-284, J. Hillis Miller Health Center, University of Florida, Gainesville, Florida 32610, USA. loped, and in 1978 a repeat penetrating keratoplasty $(7 \mathrm{~mm})$ was done in the right eye. In May 1979, when the continuous $10^{\prime} 0^{\prime}$ nylon suture was removed, a greyish-white membrane was observed in the upper $1 / 3$ of the graft endothelium. In June 1979 the visual acuity in this eye was 20/100, and the graft was hazy, with an intact epithelium. There was host vascularisation to the scar of the graft, with a prominent vessel at 10 o'clock in an area which appeared to be a fistulous tract covered by epithelium. The intraocular pressure was $10 \mathrm{mmHg}$. There was a thin whitish membrane comprising two-thirds of the posterior surface of the graft, with sharply demarked festooned edges below the optical axis. Ten days later the leading edge of this membrane had migrated further down toward the 6 o'clock position, where only a small, clear area of the graft was observed (Fig. 1, left). An $8.0 \mathrm{~mm}$ penetrating keratoplasty was performed which included the old scar and the apparent fistulous tract at 10 o'clock. The graft was clear and the visual acuity was improved to 20/30 for a period of 7 months; then ocular irritation developed with progressive clouding of the graft.

Case 2. A 68-year-old white male had a cataract extraction in his right eye 15 years ago and gradually developed bullous keratopathy. When first seen visual acuity in this eye was $20 / 400$, and the cornea 

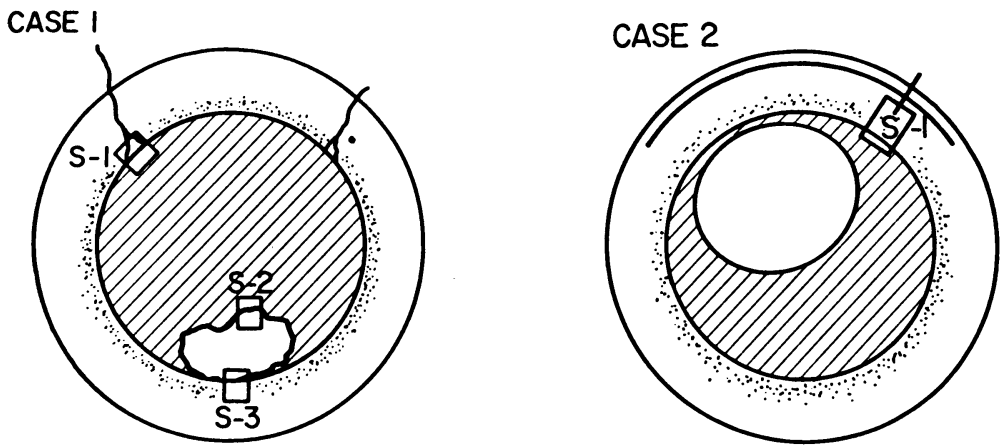

Fig. 1 Left (case 1): Diagram shows the extension of the epithelial downgrowth. Specimen $(S-1)$ corresponds to the apparent fistulous tract. Right (case 2): Clear area of the graft is from 9 to 12 o'clock. The fistulous tract $(S-1)$ of the suture from an old cataract wound is at 1.30 o'clock. Oblique lines show the area of epithelial downgrowth.

had dense haziness without vascularisation. The posterior cornea and anterior chamber could not be observed in detail. A cataract extraction scar was evident, with silk sutures still present at 10 o'clock and at 2 o'clock.

On 21 August, 1979 a $7.5 \mathrm{~mm}$ penetrating keratoplasty (interrupted 9.0 silk sutures) was performed without complications. However, 3 weeks later an area in the 3 o'clock position of the graft became hazy, with the appearance of an early graft reaction. The area of haziness moved toward the centre of the graft while the patient was on topical and systemic steroid therapy. Mild iritis was present, with an intraocular pressure of $9 \mathrm{mmHg}$. A tentative diagnosis of epithelial downgrowth was made even though no fistulous tract was found.

On 30 October, 1979 a $9.0 \mathrm{~mm}$ penetrating keratoplasty was performed. At the time of surgery an exploration of the old cataract wound revealed a fistulous tract at the 1.30 o'clock position of the corneal limbus after a silk suture was removed (Fig. 1, right). The graft was slightly decentered upwards to include the previous graft scar and the fistulous tract. Eight months postoperatively the graft was clear and visual acuity was $20 / 100$. The intraocular pressure was $18 \mathrm{mmHg}$.

\section{METHODS}

The specimens obtained at operation were fixed in $2.5 \%$ glutaraldehyde and $3 \%$ formalin with phosphate buffer ( $\mathrm{pH} \mathrm{7 \cdot 2)}$ for 90 minutes, post-fixed in cold $1 \%$ osmium tetroxide with the same buffer for 90 minutes, dehydrated in graded alcohols, and embedded in Epon. Embedded tissues were cut with a Porter-Blum MT-2 microtome and stained with toluidine blue for light microscopy and uranyl acetate and lead citrate for transmission electron microscopy (Zeiss 9S-2). Specimens for scanning electron microscopy (ETEC Omni Scan) were dehydrated in graded alcohols, dried in a critical point machine, placed on aluminium stubs, and coated with gold palladium.

\section{Results}

A diagram (Fig. 1) shows the extension of the epithelial downgrowth of case 1 and case 2 . In the first case the probable fistulous tract was located at 10 o'clock, and the clear area of the graft was at 6 o'clock. In case 2 the fistulous tract was at 1.30 o'clock near the corneal limbus and the only clear area of the graft was from 9 to 12 o'clock.

\section{LIGHT MICROSCOPY}

Case 1. Cross-sections of the specimen (Fig. 1, S-1) examined at low magnification showed the epithelial downgrowth compromising most of the corneal graft. Serial sections at the 10 o'clock area of the graft scar failed to show a fistulous tract. However, there were islands of epithelial tissue within the scar (Fig. 2). The thickness of the host cornea was about half that of the corneal transplant. Bowman's membrane was absent and the epithelium was irregular. There were fragments of Descemet's membrane incarcerated in the scar and islands of epithelium between scar tissue and strands of Descemet-like membrane material. A retrocorneal fibrous membrane was covered by a multilayered epithelium. Fig. 3a shows the advancing edge of the epithelial downgrowth (Fig. 1, S-2). In the stroma, near the Descemet's membrane, some cell infiltration was observed. The membrane had 5-6 layers of epithelium. However, the leading layer was much thicker (about $45 \mu \mathrm{m}$ ) than the trailing. Distance from the free edge of epithelial downgrowth to the advancing edge of the epithelial downgrowth over Descemet's membrane was about $200 \mu \mathrm{m}$. Flat endothelial cells were present ahead of the advancing epithelium, where histiocyte-like cells were present attached to the epithelial tissue or at the epithelial/ endothelial junction areas. On the other hand, at the 6 o'clock position (Fig. 1, S-3), near the edge of the corneal transplant, the epithelial downgrowth grew over a thin retrocorneal fibrous membrane, possibly originating from the scar. Endothelial cells were 
Fig. 2 Light microscopic section of the cornea (case 1, $S-1)$ showing the defective graft scar and islands of epithelial tissue* within the scar, new vessels, fragments of Descemet's membrane (arrows) incarcerated in the wound, and an extensive epithelial overgrowth (Epd) on the posterior surface of the cornea. ( $E P=$ epithelium,

$G=$ graft,$H=$ host, ac $=$ anterior chamber). $(\times 270)$.
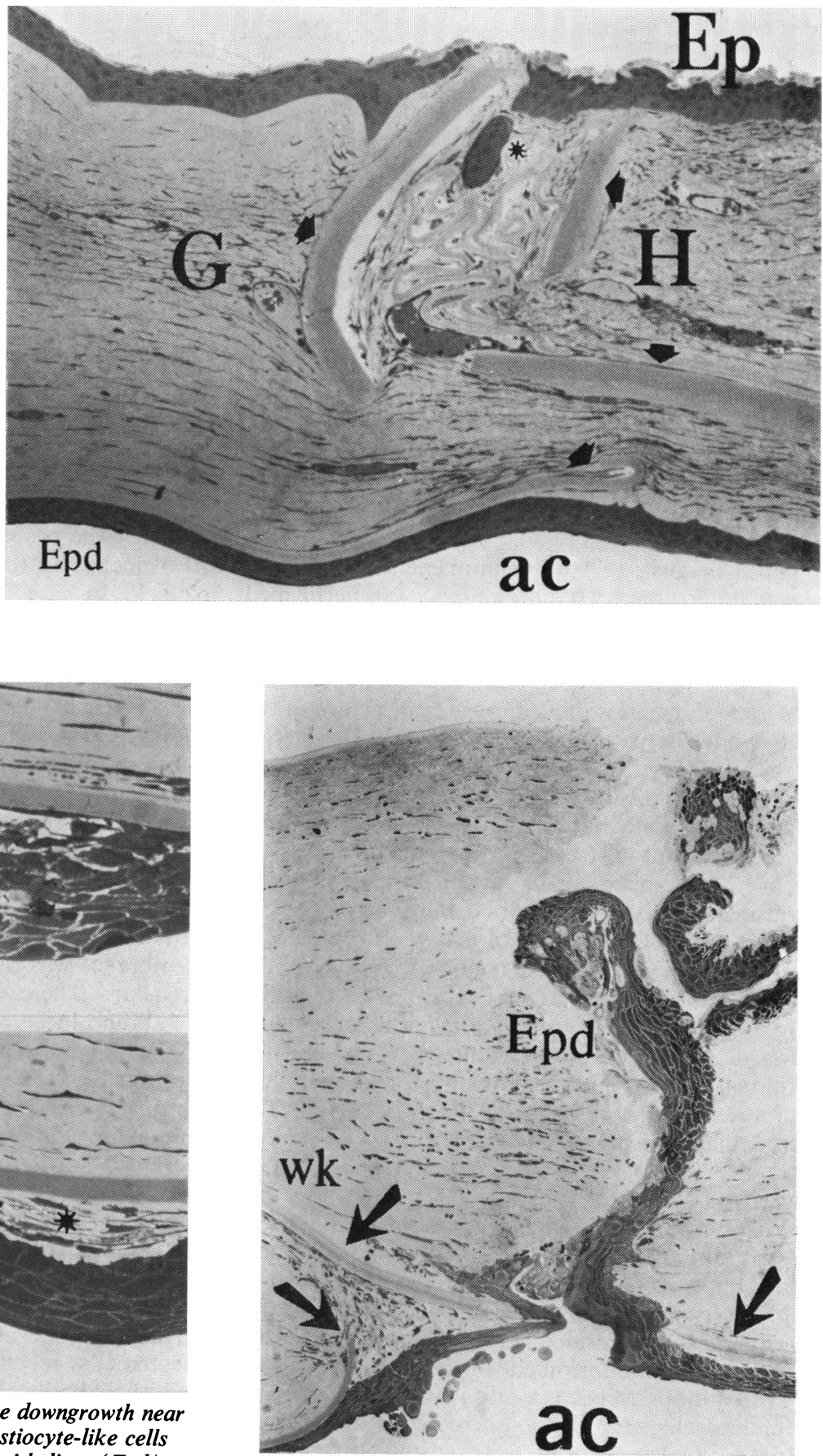

Fig. 4 Failed graft of case 2 showing a fistulous tract with epithelium (Epd) which corresponds to an old cataract suture. Wound of the first keratoplasty (WK) is left side. (Arrow = Descemet's membrane, ac=anterior chamber.) $(\times 93)$. 

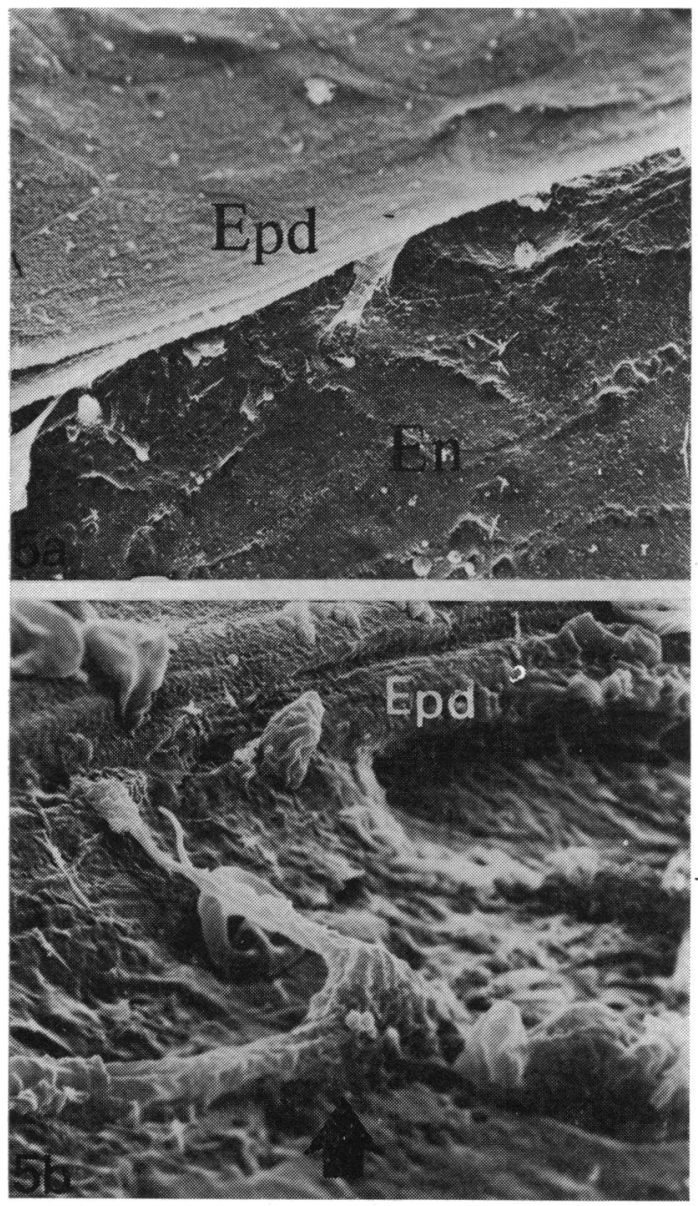

absent, and Descemet's membrane was bare ahead of the growing epithelial layer (Fig. 3b).

Case 2. No epithelial downgrowth was observed in the corneal button of the first keratoplasty. However, the specimen of the failed graft showed a fistulous tract into the anterior chamber (Fig. 4), which corresponded to an old cataract suture placed inside the cornea. Several layers of epithelium covered the suture tract and the posterior surface of the graft and the host cornea.

SCANNING ELECTRON MICROSCOPY

Scanning electron microphotographs of case 1 showed the epithelial tissue growing adjacent to the abnormal looking endothelium, which has lost its hexagonal shape (Fig. 5a). Case 2 showed that under the free advancing edge of the epithelium there were remnants of endothelial cells, histiocytes, red cells, and melanin pigment (Fig. 5b).

TRANSMISSION ELECTRON MICROSCOPY

Fig. 6 shows the advancing edge of the epithelial downgrowth over Descemet's membrane (Fig. 1, S-2). The fibroblastic endothelial cells adjacent to the

Fig. 5 (a) Advancing edge of the epithelial downgrowth over the graft endothelium of case 1 . Note that this epithelial edge is free but there are some endothelial cells (En) which lost their hexagonal shape and some cells under the epithelium (Epd). (SEM $\times 869)$. (b) Higher magnification photograph of an area of endothelium below the edge of the epithelial downgrowth (Epd) of case 2. A histiocyte (arrow) lies over remnants of endothelial cells $(S E M, \times 1603)$.

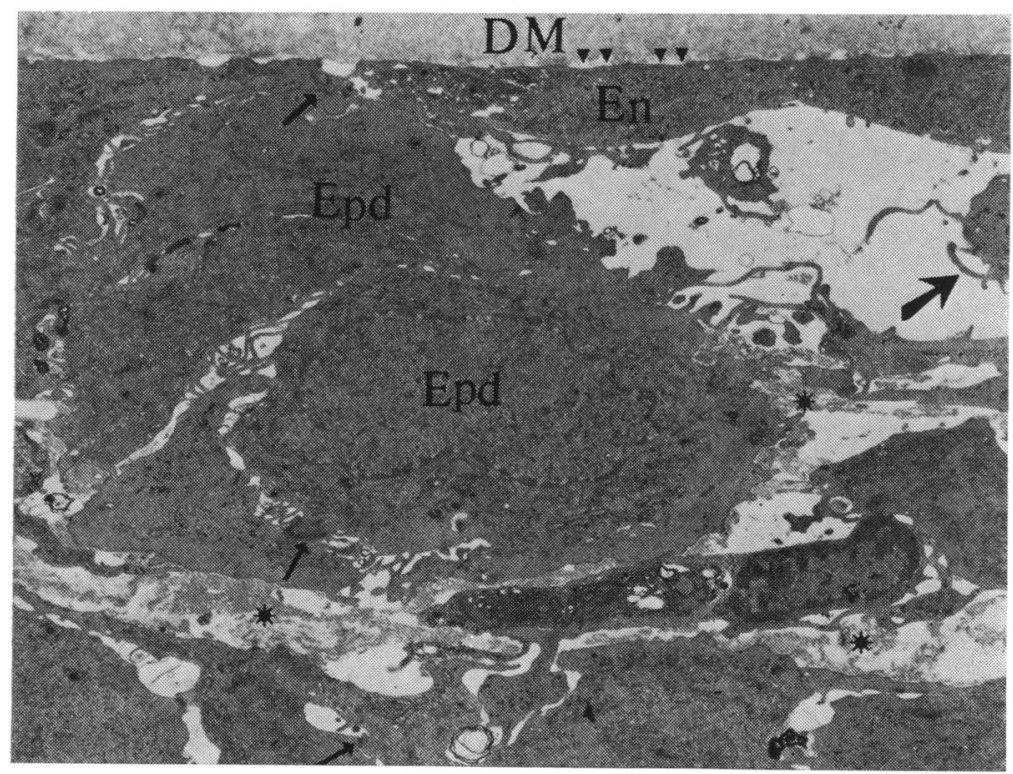

Fig. 6 TEM of the advancing edge (Fig. 1, S-2) of downgrowth epithelium (Epd). Fibroblastic endothelium cells are flat and short. Attachment between endothelium (En) and Descemet's membrane (DM) is slightly loose (double wedges, top). Fine fibrillar material* was observed in intercellular spaces of the downgrowth epithelium. (Thin arrow $=$ desmosomes, thick arrow = monocyte. $)$ $(\times 5000)$. 
Fig. 7 A more advanced area than Fig. 6. Monocyte (thick arrow) between endothelium (En) and downgrowth epithelium (Epd). Intercellular space of endothelium is separating (thin arrow) $(\times 9240)$.
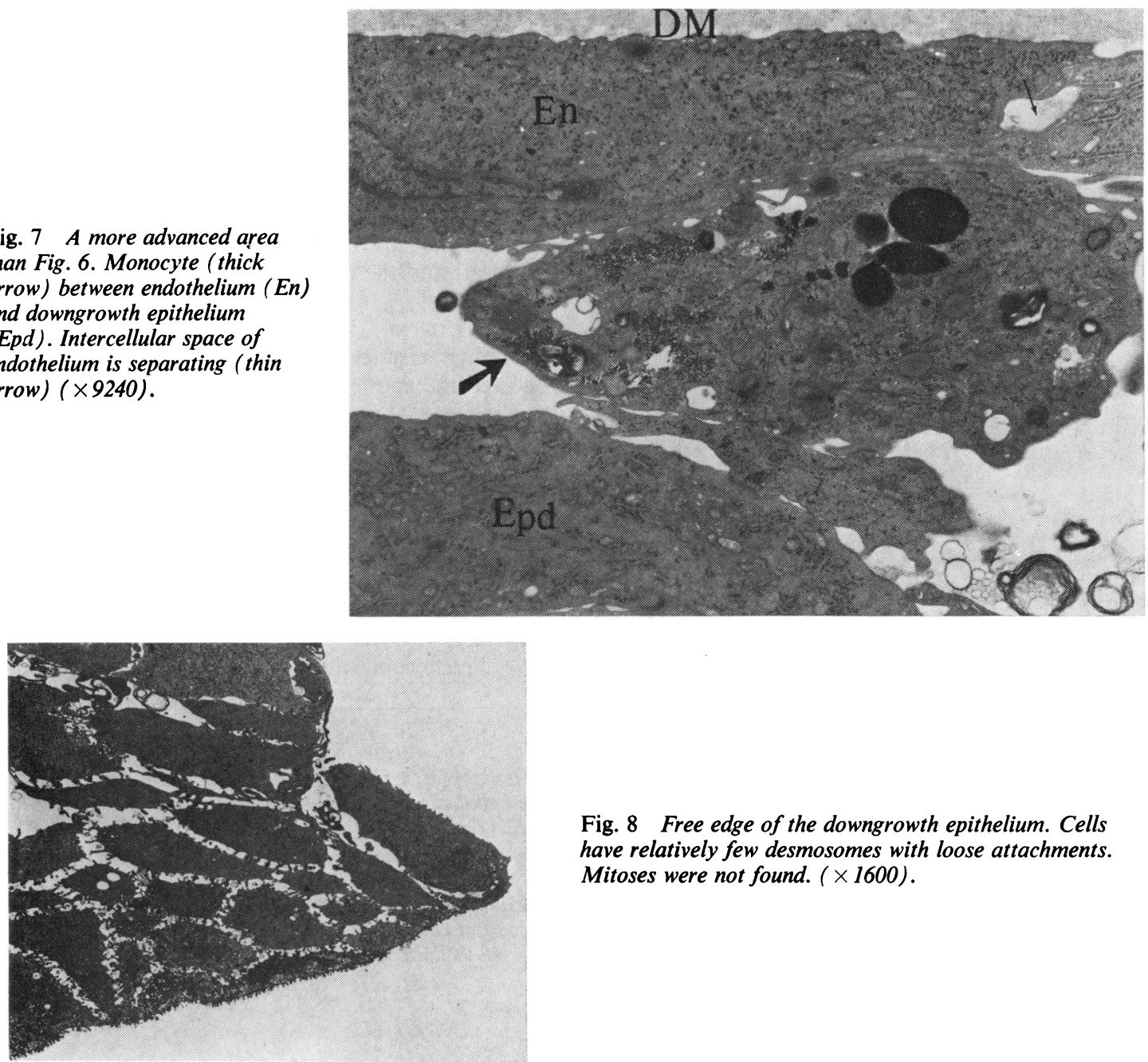

Fig. 8 Free edge of the downgrowth epithelium. Cells have relatively few desmosomes with loose attachments. Mitoses were not found. $(\times 1600)$.

Fig. 9 The well established 5 or 6 layers of downgrowth epithelium at the centre of the graft. The thickness of this layer was $1 / 4$ the thickness of the normal corneal epithelium. ( $D M=$ Descemet's membrane. EDd= downgrowth epithelium $a c=$ anterior chamber. $)(\times 4050)$.

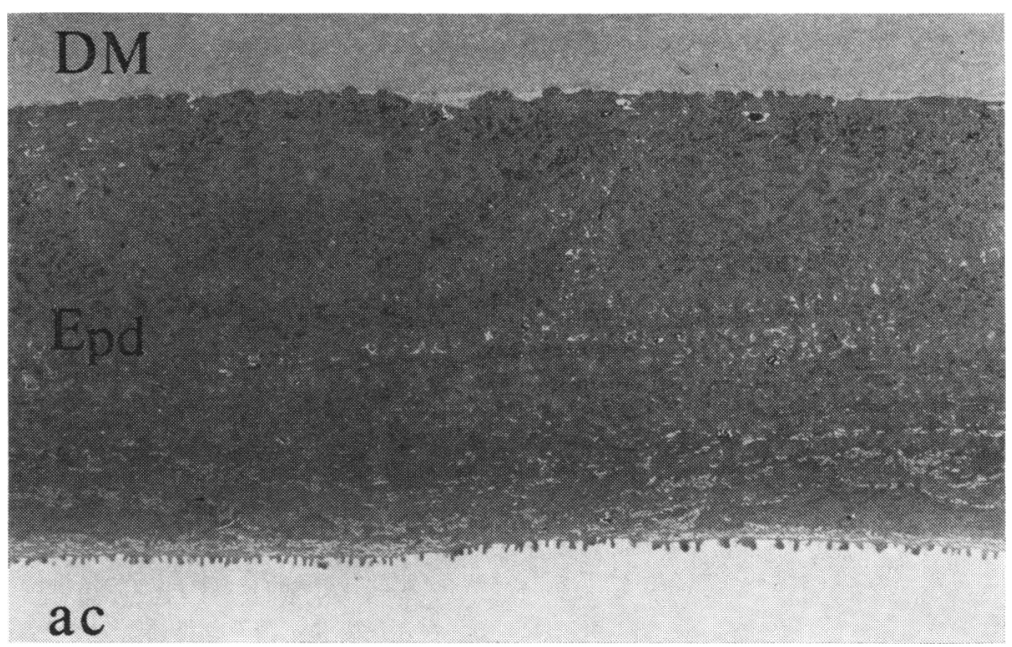




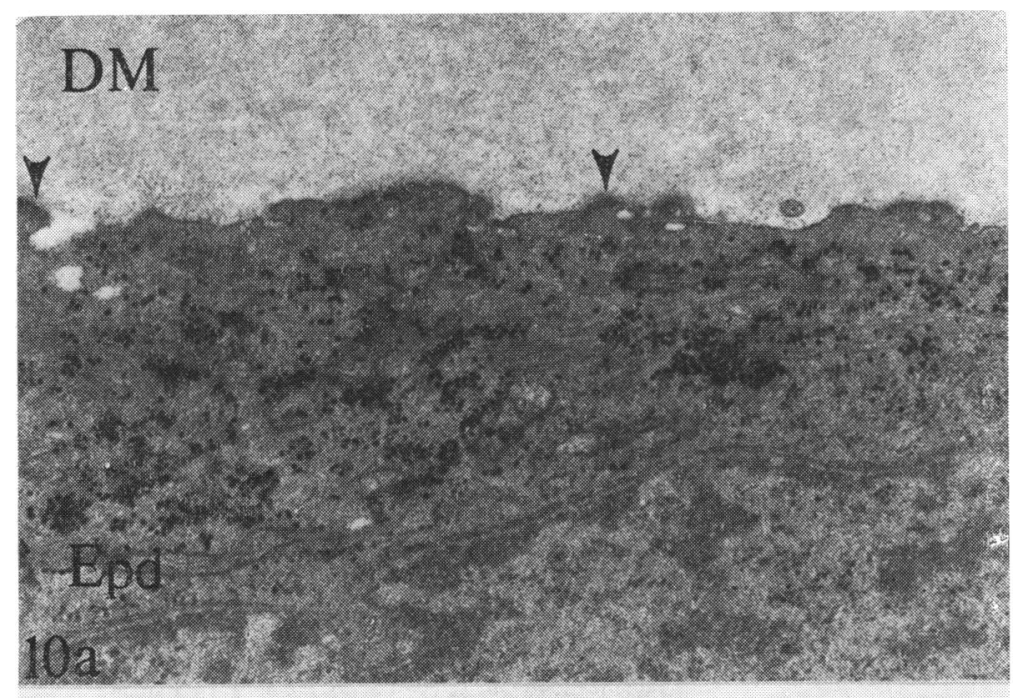

Fig. 10 (a) High magnification shows poorly developed hemidesmosomes (arrows) between Descemet's membrane (DM) and downgrowth epithelium (Epd) at the centre of the graft. $(\times 26450)$. (b) Relatively well developed Rfm hemidesmosomes (small arrows) and basement membrane (thick arrow) are seen between downgrowth epithelium (Epd) and retrocorneal fibrous membrane ( $R \mathrm{fm}$ ) near the host graft junction. $(\times 26450)$.

epithelium were flat and short and had intracytoplasmic vacuoles, few mitochondria, prominent or rough endoplastic reticulum, and loose attachment to Descemet's membrane. The intercellular space of endothelium was often separated. Fine fibrillar material and basement membrane-like material (Fig. 12b) were observed in the intercellular spaces of the downgrowth epithelium.

At more advanced areas than Fig. 6 shows between the epithelium and the endothelium there were monocytes or a few lymphocytes attached to either tissue (Fig. 7). In their cytoplasma lysosomes and some debris were observed. Fig. 8 shows the free edge of the downgrowth epithelium. Epithelial cells had prominent cytoplasmic processes, tonofilaments, and prominent free ribosomes, but mitosis was not found. Cells had relatively few desmosomes with loose attachments between them and microvilli on its anterior chamber surface.

The well established epithelial downgrowth in the centre of the corneal transplant was 5 to 6 layers thick (Fig. 9). The thickness of this layer was about $11 \cdot 3 \mu \mathrm{m}$ or roughly $1 / 4$ of the thickness of the normal corneal epithelium. The cells had dense cytoplasm and prominent free ribosomes. Cells facing the anterior chamber had tight junctions. At the centre of the graft subepithelial fine collagen fibrils were sometimes observed between Descemet's membrane and endothelium. At this area hemidesmosomes were not well developed (Fig. 10a). On the other 
Fig. 11 Epithelium facing the anterior chamber has microvilli with fine filamentous strands. Double membrane-like material (arrows) is at about 0.4um over the anterior chamber $(a c)$. $(\times 61650)$.

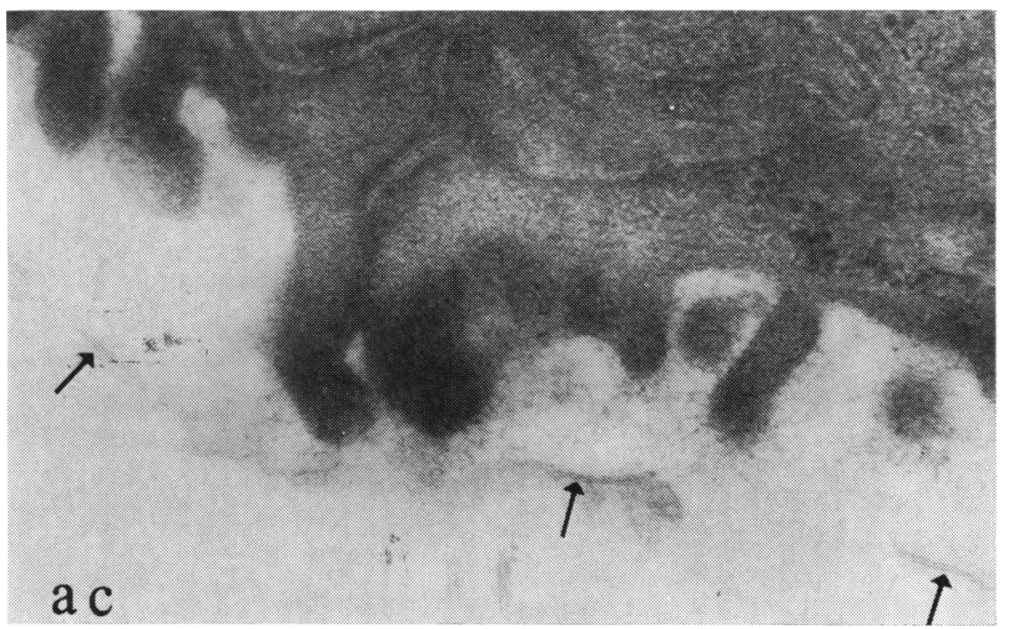

hand relatively well developed hemidesmosomes and basement membrane were observed between retrocorneal fibrous membrane and the downgrowth epithelium near the host-graft junction (Fig. 10a, Case 1, S-1). In this area, where the retrocorneal fibrous membrane was thicker, more well developed hemidesmosomes were observed with a basement membrane. As with the cells in the advancing edge the well established epithelium facing the anterior chamber had microvilli with fine filamentous strands as in normal epithelium (Fig. 11). In some areas, at about $0.4 \mu \mathrm{m}$ over the anterior chamber surface of the downgrowth epithelium, an interrupted double membrane-like material was observed. These cells also had prominent vesicles and glycogen granules in their cytoplasma. Near the host-graft junction (Fig. 1, case 1, S-3) endothelium was already absent, and cell debris was present ahead of the growing epithelium (Fig. 12a). Fibrillar material was present over epithelial cells with deposits of basement membrane-like material (Fig. 12b). Undeveloped hemidesmosome-like material was observed.

No goblet cell was found in any specimens.

\section{Discussion}

The ultrastructural findings of the downgrowth epithelium of our cases resembled normal corneal epithelium rather than normal conjunctival epithelium. ${ }^{8} 9$

Comparing the downgrowth epithelium of this case with true corneal epithelium, we found that ultrastructural features were similar but differences seen were as follows: the thickness of the epithelial layer was $1 / 4$ of the thickness of the normal; the shape of the downgrowth epithelium was flatter. The cytoplasma was more dense. The basal cell layer (attached to the Descemet's membrane) of the down-

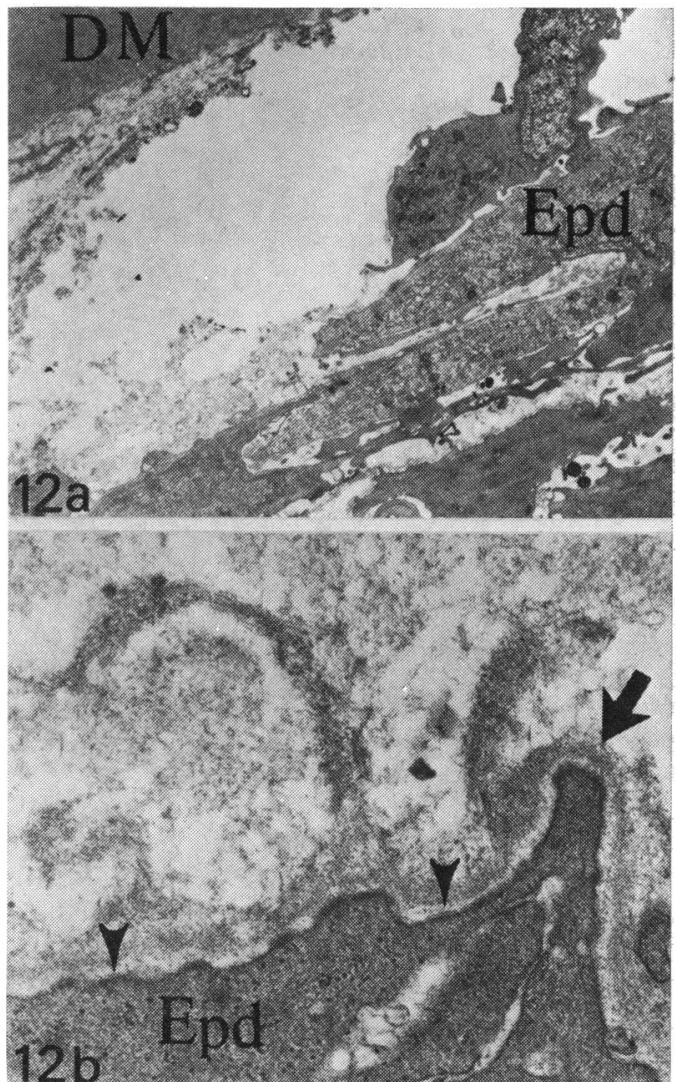

Fig. 12 Advancing edge of epithelial downgrowth (Epd) near the host-graft junction (Fig. 1, case 1,S-3), endothelium is absent, and cell debris is present. (DM=Descemet's membrane $).(\times 3972)$. (b) Basementmembrane-like material (thick arrow) and undeveloped hemidesmosomes (small arrows) around the fine fibrillar material between the downgrowth epithelium (Epd) are being produced by downgrowth epithelium. $(\times 25920)$. 
growth epithelium had many more free ribosomes. The surface cell layer had more glycogen granules than normal. The downgrowth epithelium had few desmosomes, especially hemidesmosomes between Descemet's membrane and the basal layer of the downgrowth epithelium. They were poorly developed at the centre of the graft. The downgrowth epithelium also had an interrupted double membrane-like material on the anterior chamber surface.

Three electron microscopic studies of epithelial downgrowth following cataract extraction have been reported. ${ }^{10-12}$ Iwamoto et al. ${ }^{10}$ reported that subepithelial connective tissue at varying stages of development was present between the downgrowth epithelium and Descemet's membrane. In our case near the host-graft junction results were the same as Iwamoto's. However, centrally the development of the subepithelial connective tissue was minimal or completely absent (Fig. 10a). This suggests that subepithelial fibrous tissue may have originated from the scar, but the possibility also exists that it may have been produced by epithelial tissue. We can assume that Iwamoto's specimen was taken nearer the incision than ours.

The hemidesmosomes and basement membrane connecting the epithelial downgrowth to the retrocorneal fibrous membrane were well developed (Fig. 10b). However, undeveloped hemidesmosomes were observed between Descemet's membrane and downgrowth epithelium, especially near the advancing edge (Fig. 10a). Histologically it appears that attachment of these tissues seemed to be not as strong as normal epithelium attached to Bowman's membrane. The clinical tendency of recurrent epithelial downgrowth ${ }^{1314}$ might be supported by this fact.

An interrupted double-membrane-like material about $0.4 \mu \mathrm{m}$ thick on the anterior chamber surface of the downgrowth epithelium was observed (Fig. 11). This membrane-like material looked like an osmiophilic (lipid) layer as secreted in the normal epithelium. ${ }^{8}$ Hogan et al. ${ }^{8}$ observed single-membranelike material on the surface of the normal epithelium but not double-membrane-like material. The reason for this difference is unknown. In any event the surface cells of the downgrowth epithelium in the anterior chamber appear to secrete this layer, as does the normal epithelium.

The difference in histological features between the downgrowth epithelium in the anterior chamber and normal corneal epithelium on the Bowman's membrane might depend on circumstances such as nutrition (including oxygen), intraocular pressure, osmolar pressure, temperature, and so on.

The endothelium near the incision (host-graft junction) (Figs. 3b, 12a) had more damage than at the centre of the graft (Figs. 3a, 6), a well known fact in corneal grafts 1516 and after cataract extraction. ${ }^{17}$ It has been suggested that such alteration is necessary for the growing of the epithelial downgrowth. ${ }^{78}$ This is the first report to show the advancing edge of the invading epithelium near the centre of the graft, where fibroblastic endothelium was degenerated but was still present and appeared to be overrun by the former (Fig. 6). Clinically the speed of progression of the epithelial downgrowth near the host-graft junction was faster than that of the centre of the graft. These findings indicate that the absence of endothelium is not essential to the progression of the epithelium but facilitates the advance of the downgrowth.

Terry et al. ${ }^{18}$ reported that presence of endothelium inhibits epithelial downgrowth. It has been shown in vitro that endothelial cells and epithelial cells growing in the same area show contact inhibition when they meet. ${ }^{19}{ }^{20}$ In vivo, however, conditions are not quite the same, and contact inhibition does not occur. Near the advancing edge monocytes and a few lymphocytes were observed. Possibly the mechanical destruction of endothelial cells by the fast growing epithelium and the effect of inflammatory cells might help its progression.

Case 1 had a keratoplasty and a cataract extraction in the same eye 16 and 9 years ago respectively. Shortly after a repeat keratoplasty epithelial invasion of the posterior cornea developed. Clinically and histologically the host-graft junction at the 10 o'clock position appeared to have a fistula from which the corneal epithelium had invaded the posterior cornea. Therefore the epithelial downgrowth in Case 1 was apparently caused by placing a graft on a thin host cornea, by incomplete host cutting with wound incarceration of Descemet's membrane, and tight and probably perforating sutures. In case 2 the corneal button of the first keratoplasty did not have downgrowth epithelium histologically, so it may have been caused by excessive tension on the 15-year-old cataract wound when the graft was sutured. It has not been reported that epithelial downgrowth occurred through a fistulous cataract suture tract after penetrating keratoplasty. Christensen, ${ }^{21} \mathrm{Kara},{ }^{22}$ Dunnington and Regan $^{23}$ and Dunnington ${ }^{24}$ reported that excessively tight sutures in cataract wounds caused stromal necrosis and encouraged epithelial growth into the anterior chamber. Since in both cases epithelialisation seemed to occur along suture tracts in very thin or diseased corneas, these cases point to the importance of proper tension and depth of sutures. ${ }^{2} 618$ 23-26 Care should be taken in the case of a patient who has had previous intraocular surgery. Good alignment of Descemet's membrane between the host and graft 
at time of surgery, especially when there are differences in corneal thickness, is important. Finally, damage to the donor endothelium must be avoided.

We thank Maruja McNiece for her help in preparing this manuscript.

This work was supported in part by USPHS Grant EY00415, National Eye Institute, National Institutes of Health, Bethesda, Maryland, and by the North Florida Lions Eye Bank for Restoring Sight, Inc.

\section{References}

1 Kitagawa H, Komori Y, Hara J. A case of retrocorneal membrane with an epithelial cyst following perforating keratoplasty. Folia Ophthalmol Jap 1965; 16: 424-7.

2 Mazow ML, Stephens RW. An usual complication after keratoplasty. Surv Ophthalmol 1966; 11: 205-8.

3 Leibowitz HM, Elliott JH, Boruchoff SA. Epithelization of the anterior chamber following penetrating keratoplasty. Arch Ophthalmol 1967; 78: 613-7.

4 Kurz GH, D'Amico RA. Histopathology of corneal graft failures. Am J Ophthalmol 1968; 66: 184-99.

5 Bennet T, D'Amico RA. Epithelial inclusion cyst of iris after keratoplasty. Am J Ophthalmol 1974; 77: 87-9.

6 Arentsen JJ, Morgan B, Green WR. Changing indications for keratoplasty. Am J Ophthalmol 1976; 81: 313-8.

7 Sugar, Meyer RA, Hood CI. Epithelial downgrowth following penetrating keratoplasty in the aphake. Arch Ophthalmol 1977; 95: 464-7.

8 Hogan MJ, Alvarado JA, Weddell JE. Histology of the Human Eye, An Atlas and Textbook. Philadelphia: W. B. Saunders Company, 1971: 75-83.

9 Kuwabara T. Fine Structure of the Eye. 2nd ed. Boston: Security-Columbian, 1970: 4-14.

10 Iwamoto T, Srinivasan BD, Devoe AG. Electron microscopy of epithelial downgrowth. Ann Ophthalmol 1977; 9: $1095-110$

11 Jensen P, Minckler DS, Chandler JW. Epithelial ingrowth. Arch Ophthalmol 1977; 95: 837-42.
12 Ghosh M, McClloch C. Epithelial downgrowth following cataract extraction. Can J Ophthalmol 1979; 14: 126-36.

13 Harbin TS Jr, Maumenee AE. Epithelial downgrowth after surgery for epithelial cyst. Am J Ophthalmol 1974; 78: $1-4$.

14 Maumenee AE. Treatment of epithelial downgrowth and intraocular fistula following cataract extraction. Trans Am Ophthalmol Soc 1964; 62: 153-66.

15 Brightbill FS, Polack FM. A comparison of two methods for cutting donor corneal buttons. Am J Ophthalmol 1973; 75: 500-6.

16 Polack FM. The endothelium of failed corneal grafts. Am J Ophthalmol 1975; 79: 251-61.

17 Rao GN, Shaw EL, Arthur E, Aquavella JV. Morphological appearance of the healing corneal endothelium. Arch Ophthalmol 1978; 96: 2027-30.

18 Terry TL, Chisholm JF, Schonberg AL. Studies on surface-epithelium invasion of the anterior segment of the eye. Am J Ophthalmol 1939; 22: 1083-110.

19 Cameron JD, Flaxman BA, Yanoff M. In vitro studies of corneal wound healing, epithelial-endothelial interactions. Invest Ophthalmol Visual Sci 1974; 13: 575-9.

20 Yanoff M, Cameron JD. Human cornea organ culture: epithelial-endothelial interactions. Invest Ophthalmol Visual Sci 1977; 16: 269-73.

21 Christensen L. Epithelization of the anterior chamber. Trans Am Ophthalmol Soc 1960; 58: 284-300.

22 Kara GB. Histologic appearance of an eye four days after cataract extraction. Arch Ophthalmol 1953; 49: 285-92.

23 Dunnington JH, Regan EF. The effect of sutures and of thrombin upon ocular wound healing. Am J Ophthalmol 1952; 35: 167-77.

24 Dunnington JH. Ocular wound healing with particular reference to the cataract incision. Arch Ophthalmol 1956; 56: $639-59$.

25 Dunnington JH. Healing of incision for cataract extraction. Am J Ophthalmol 1951; 34: 36-45.

26 Maumenee AE. Epithelial invasion of the anterior chamber retinal detachment corneal edema anterior chamber hemorrhage changes in the macula. Trans Am Acad Ophthalmol Otolaryngol 1957; 61 : 51-68. 